\title{
Trajets et dérives autour d'une prison de femmes
}

\author{
Manuela Ivone Cunha \\ Universidade do Minho (Portugal)
}

\begin{abstract}
Résumé
La prison constitue l'un de ces contextes de recherche qui, de façon immédiate et consensuelle, apparaissent concernés par la notion de " terrain miné », notamment parce que ce contexte est par ailleurs pré-défini comme un " problème social ", et que la formulation d'une recherche-diagnostic ne va pas toujours de soi. Mais il reste d'autres mines, des pièges et des questions théoriques et méthodologiques non identifiés au départ, qui ne sont pas d'une nature différente de ceux qui existent virtuellement sur tous les terrains. C'est de ce genre de mines, qu'il est question ici. L'article expose une situation de retour sur le terrain dix ans après et s'interroge sur le sens des difficultés qui ont marqué la progression d'une enquête plus récente. Sont ainsi mis en évidence les profonds changements qui ont affecté un établissement carcéral féminin au Portugal, et est relancée la discussion sur le statut théorique de la prison et de la frontière la séparant des mondes extérieurs.
\end{abstract}

Mots-clefs : Prison. Femme. Drogue. Exclusion. Portugal.

Manuela Ivone Cunha

IDEMEC

5, rue du Château de l’Horloge

13094 Aix-en-Provence

micunha@ics.uminho.pt

Tous les terrains, en tant que terrains de recherche, sont potentiellement minés par des circonstances inhérentes aux contextes eux-mêmes ou au regard que l'on porte sur eux, sans parler des inévitables équivoques et malentendus qui marquent le cheminement de toute enquête. Pourtant certains, comme c'est le cas des prisons, semblent d'emblée plus minés que d'autres et méritent ce qualificatif dès le départ, et de façon presque auto-justificative. Il est néanmoins vrai que ces contextes convoquent souvent des contenus plus saillants et immédiats qui, entre autres, s'abritent sous la notion de "terrain miné ». Cette notion peut, tout d'abord, correspondre au fait qu'un terrain est généralement considéré comme un problème social. Sont alors attendus de la part du chercheur un diagnostic et ses conséquences, c'est-à-dire des propositions pour intervenir sur ce problème. Il peut ensuite y avoir le fait qu'un terrain est déjà approprié par une variété de discours forts - communs ou spécialisés - qui peuvent ainsi contaminer au départ ce qui pouvait ou aurait pu constituer un autre regard. Un terrain, enfin, peut être miné parce qu'il est simplement, d'un point de vue humain, difficile et parfois même dramatique.

Le terrain sur lequel j'ai travaillé, une prison de femmes, au Portugal, est certainement, dans tous ces sens, miné. J'aimerais pourtant - ayant par ailleurs entamé une opération de déminage en abordant spécifiquement certaines questions que je viens d'énoncer [Cunha, 1991] - exposer quelques lignes de ma recherche sous un autre angle, et ce au travers de deux thèmes : le retour sur le terrain (j’ai récemment séjourné dans la prison où j'avais réalisé une première enquête dix ans auparavant) et, d'autre part, la difficulté que j’ai éprouvée en 
réalisant l'enquête actuelle, à la différence de ma première expérience. Pour reprendre la métaphore, il sera donc question d'autres « mines », mais au sens faible du terme. Il s'agit de celles qui investissent virtuellement tous les terrains. Dans ce sens, le « terrain prison » n'est pas a priori plus « miné » que d'autres, et le qualifier ainsi risquerait de le réifier en tant que réalité essentiellement problématique, en dehors de toute construction qui en serait faite par le chercheur.

J'espère éviter deux tendances en abordant ces thèmes : d'une part, le ton " confessionnel » (j'emploie ce mot sans sa connotation religieuse), qui marque parfois les récits d'arrivée sur le terrain et qui peut être gratuit si l'expérience personnelle n'a pas de portée visible pour comprendre l'objet; d'autre part, je vais essayer de ne pas trop revêtir la peau de l'ethnologue " héros ", rôle vers lequel souvent on se sent subtilement poussé dès que l'on travaille sur un terrain miné ou qui est perçu comme tel. Ces deux pièges semblent toujours accompagner de telles situations de recherche.

Plusieurs changements importants ont marqué ce retour sur le terrain. Des changements institutionnels, telle l'organisation de la prison, d'abord. D'une prison de type ferme rurale, avec une gestion domestique et presque familiale (et je pense notamment à cette assistante sociale qui parlait avec nostalgie de l'ancienne directrice comme d' "une mère qui accueillait tout le monde »), on passe à une institution véritablement bureaucratique, avec des départements très spécialisés (dont certains ne dépendent même plus de la direction de la prison) et au personnel professionnalisé (les membres du personnel technique, beaucoup plus jeunes, ont désormais tous, à une exception près, fait des études supérieures, ce qui n'était pas le cas auparavant). Deuxièmement, on observe des transformations dans le volume et le type de population incarcérée. On est passé d’à peine deux cents prisonnières en 1987 à presque neuf cents aujourd'hui, une augmentation qui a été causée plus par le transfert de détenues depuis une autre prison, entre temps fermée, que par l'accroissement de la criminalité féminine ${ }^{1}$. Il existait autrefois une relative diversité de types de délits, avec néanmoins une majorité d'entre eux perpétrés contre les biens - chèques sans provision, escroquerie, vol. Aujourd'hui, la plupart des crimes de cette sorte sont associés à la toxicomanie (63\% des cas), et les infractions à la législation sur les stupéfiants constituent à présent l'écrasante majorité des motifs de détention (76\%). Sur cette population, $88 \%$ des femmes sont ainsi emprisonnées pour des infractions liées directement ou indirectement à la drogue ${ }^{2}$, situation qui d'ailleurs est loin d'être unique dans les prisons portugaises, tant masculines que féminines. La drogue semblant donc constituer un facteur de relative uniformisation du profil pénal des prisonnières, le profil sociologique paraît lui aussi plus unifié par deux caractéristiques : d'une part une certaine paupérisation, constatable dans des trajectoires de vie presque dickensiennes et qui concerne non seulement le capital économique mais aussi les capitaux sociaux, symboliques et scolaires ; et, d'autre part, la provenance systématique de cette population de quartiers populaires en difficulté et de minorités ethniques stigmatisées. Je reviendrai plus loin sur ce point.

Ce nivellement par le bas d'une partie importante des détenues creusait encore plus la distance sociologique qui me séparait d'elles. Vue autrefois comme une jeune étudiante, célibataire, je me retrouvais avec un autre statut, un autre capital social et symbolique. Celuici se traduisait par exemple en marques comme le titre de "docteur » (dont l'usage au Portugal dépend simplement de l'obtention d'une licence, et avec lequel on m'a présentée aux détenues) et très probablement, mais de façon plus subtile, en d'autres indicateurs incorporés dans des postures et des façons de parler. Dix ans d'enseignement ont peut-être laissé des traces dans la modulation de mon oralité, par exemple. La distance sociale entre la 
plupart des détenues et moi s'était donc élargie et, au début de la nouvelle enquête, je voyais dans cet éloignement la cause de la difficulté que j'éprouvais à établir un rapport de proximité avec elles. Mais, d'un autre côté, ce fossé sociologique était en quelque sorte comblé à un autre niveau : en écoutant leurs histoires de vie et les circonstances du délit qui les avaient conduites en prison, je ressentais constamment qu'à leur place, dans leurs contextes de vie, j'aurais peut-être fait de même. Pendant mon premier séjour, ce type d'identification n'avait au contraire été que très ponctuel. Aujourd'hui, les délits des femmes de cette prison étaient liés beaucoup plus directement à leur marginalisation structurelle, et il me semblait qu'avoir choisi de ne pas commettre de crime eût été presque héroïque de leur part. Qui plus est, pour la première fois, cette identification avec les détenues était manifeste aussi de la part du personnel pénitentiaire, ce qui allait me permettre d'ailleurs de comprendre quelques logiques du fonctionnement de ses membres et des représentations qu'ils ont des détenues.

Il est acquis désormais qu'une enquête ethnographique n'est pas définitive et atemporelle, mais au contraire sélective et contingente notamment à une relation interpersonnelle spécifique et à un moment historique particulier [Kenna, 1992 ; Okely, 1992]. Les retours sur le terrain permettent justement de situer de façon plus nette les enquêtes précédentes dans une étape d'évolution d'un contexte. J'ai déjà sommairement rendu compte des principaux aspects de la transformation de cette prison. Or, après une première analyse de mes données de terrain, je peux déjà prévoir des conclusions très divergentes de celles de ma précédente enquête. Cela est-il dû au fait que j'ai eu par hasard l'occasion d'assister à la fin d'un cycle de l'institution et au début d'un autre ? Ou, pour poser les choses de façon très schématique, les éléments centraux et des tendances importantes que je peux aujourd'hui dégager étaientils déjà là lors de ma première enquête, mais je ne les avais pas vus ? C'est-à-dire que j'aurais en effet pu ne pas les voir en raison, soit du type de relations personnelles que j'avais établies avec les détenues, soit des lunettes théoriques dont je m’étais munie qui pouvaient orienter mon regard dans une direction et l'éloigner d'une autre. Il est vrai qu'une enquête est conduite dans une étape non seulement du cycle de vie du chercheur, mais aussi de sa maturation intellectuelle et de son parcours théorique.

Or j’ai eu la surprise - extrêmement rassurante même pour quelqu'un qui avait déjà appris à accepter le caractère contingent des résultats d'une enquête ethnographique - de constater qu'une frange minoritaire de détenues développe à présent exactement les mêmes discours, représentations, pratiques et formes de sociabilité qui étaient saillants il y a dix ans dans la majorité de la population carcérale. L'actuel groupe de détenues (qui n’est pas bien sûr le même qu'auparavant) correspond, de façon presque figée, aux descriptions et analyses que j'ai faites lors de ma première enquête. Le retour sur le terrain a produit un effet de contrôle rétrospectif inattendu, mais qui est valable aussi pour la présente recherche. Brièvement, si je vois à peu près avec les mêmes lunettes mais que ce que je vois est très différent, c'est que ce sont principalement des transformations externes à mon parcours personnel qui produisent des conclusions divergentes.

Je reviens néanmoins sur le caractère intersubjectif de l'enquête ethnographique, et les contingences qui en découlent. J'ai déjà évoqué la difficulté que j'ai ressenti à dépasser un rapport superficiel avec les détenues, qui ont, d'une façon générale, mis longtemps à abandonner la réserve qu'elles me manifestaient. Lors de mon premier séjour, ce qui était intrigant, c'était justement le contraire, c'est-à-dire l'aisance et la rapidité avec lesquelles ce rapport se construisait, souvent d'ailleurs sur leur initiative. Comme je l'ai déjà indiqué, j'ai commencé par attribuer mes difficultés actuelles à établir une relation de confiance au fossé 
qui s’était creusé entre les détenues et moi. Bien évidemment, cette attribution n’expliquait rien en elle-même. S'il en était ainsi, une bonne partie de la recherche ethnologique n'aurait pas été possible, vu la distance sociale et culturelle qui souvent sépare les ethnologues et les populations qui les accueillent.

Ce n'est que plus tard que j'ai pris conscience qu'était toujours valide ce que j'avais écrit à propos de mon premier séjour, à savoir que "mon rapport avec les détenues était inextricablement lié avec la nature de leurs relations interpersonnelles, et qu'il ouvrait sur les enjeux identitaires en ouvre " [Cunha, 1994: 11]. Lors de ma première enquête, le rapport de rapide proximité que les prisonnières avaient établi avec moi traduisait dans une certaine mesure la distance qui existait entre elles. Cette distance se manifestait dans leurs sociabilités atomisées et dans leurs efforts de déqualification de co-détenues, à propos de qui étaient reproduits des discours très stigmatisants. Le lien qu'elles avaient établi avec moi semblait leur permettre de conjurer l'identité déviante que l'incarcération attestait et qu'elles projetaient sur les autres.

Aujourd'hui, et sauf pour cette minorité que j'ai mentionnée, les enjeux sociaux et identitaires ne sont pas les mêmes. J’ai déjà fait référence au fait qu’à présent beaucoup de détenues viennent des mêmes quartiers fragilisés ou de certaines minorités ethniques. Or, non seulement beaucoup d'entre elles se connaissaient déjà avant l'incarcération, mais elles sont aussi unies par des liens de parenté : tantes, cousines, sœurs, belles-sœurs, mères, grandmères, belles-mères. Et souvent leurs très proches parents masculins sont aussi incarcérés dans d'autres établissements. Les raisons de ce fait sont diverses. Soit les forces de police sont beaucoup plus proactives à l'égard de certaines catégories socio-spatiales et ethniques, ce qui augmente la probabilité d'arrestation pour chaque membre de ces groupes, soit le commerce de détail de drogues, le plus exposé, se développe sur des réseaux vicinaux et familiaux: l'économie illégale est peu anomique; elle repose plutôt sur des sociabilités traditionnelles relativement structurées ${ }^{3}$. Enfin, il n'est pas rare, quand la police trouve des stupéfiants dans une maison lors d'une fouille, que toutes les personnes présentes, et parfois même les résidents absents, soient arrêtés préventivement.

On peut déjà avancer ici un premier élément caractérisant cet univers. Ces réseaux d'interconnaissance pré-carcérale placent la prison en continuité plus qu'en rupture avec l'extérieur. La stigmatisation qu'elle représentait autrefois s'institue à présent à l'amont de la détention. Elle s'installe déjà par le fait d'appartenir à des minorités ou à des quartiers connotés médiatiquement avec la drogue, et elle atteste d'une marginalisation plus structurelle que ponctuelle. Peut-être que les détenues me voient déjà trop éloignée et font preuve de réalisme en n'aspirant même plus symboliquement à une légitimation identitaire en s’approchant de moi. Si la stigmatisation est plutôt pré-carcérale, la prison s’est " banalisée » dans l'horizon social immédiat de ces femmes. La plupart ont des voisins ou des membres de leur famille qui sont ou ont été à un moment ou à un autre incarcérés. Des visiteurs viennent à la prison ensemble en profitant de la voiture d'un voisin qui vient aussi rendre visite à une prisonnière. Les détenues savent donc qu'en sortant elles ne seront pas inévitablement regardées de travers. Plus que banalisée, la prison s'est d'une certaine façon " normalisée ». Quand j'essayais d'éviter leurs visages en les photographiant, les détenues insistaient souvent pour que je les prenne de face et prenaient une pose qui affichait un radieux sourire. L'une d'entre elles m'a même demandé une photo, car elle en avait pris une de chaque prison dans laquelle soit elle avait été incarcérée, soit où elle avait rendu visite à quelqu'un, mais à cette collection manquait une image faite à Tires. 
La prison est donc une réalité très présente à l'amont de la détention et se trouve déjà incorporée dans le quotidien, avec une dilution de la frontière symbolique qu'autrefois elle représentait. Mais il y a aussi d'autres frontières qui sont devenues perméables. Pendant ma première enquête, les lignes de démarcation entre les détenues proliféraient dans la tentative, que j'ai déjà évoquée, d’instituer une distance sociale et symbolique avec les co-prisonnières. C'était le cas en plusieurs domaines, comme, par exemple, celui du crime qui avait causé la condamnation. Une détenue considérait son délit justifiable et ponctuel, tandis que ceux des autres découlaient de leur nature ou de leur mentalité criminelles. Et, bien sûr, la hiérarchisation de la gravité des délits variait selon l'auteur de la classification: les trafiquantes consommatrices se déculpabilisaient avec leur toxicomanie, une excuse qu'elle refusaient aux trafiquantes qui n'étaient pas des consommatrices; celles-ci se disaient réhabilitées et réhabilitables, au contraire des premières, commandées par leur compulsion ; toutes dénigraient les homicides qui, à leur tour, disaient avoir agi par désespoir et non par avidité, et sans vivre du crime; et, enfin, si, en comparaison, les condamnées pour escroquerie ou vol considéraient leur délit inoffensif, elles étaient regardées par les autres comme structurellement tricheuses et cleptomanes.

Or, à présent, outre que la variété des délits est proportionnellement beaucoup plus réduite, même les crimes contre la propriété sont associés à la toxicomanie. "On est toutes là pour drogue », me disait une détenue ; " on est toutes ensemble avec les criminelles », m'affirmait une autre, entendant par " criminelles » celles qui avaient été condamnées pour homicide. L'homicide est en effet globalement le seul crime qui institue désormais une frontière tangible entre les détenues, pouvant constituer un instrument de déqualification dans les deux sens (le recel, comme on le verra plus loin, est un cas à part, qu'il convient d'analyser dans un contexte extra-carcéral). Néanmoins, il n'est pas très présent en tant que tel dans la sociabilité quotidienne, et de toute façon la proportion de détenues condamnées pour ce crime est très réduite. Mais ce n’est pas seulement la convergence de types ou de motifs de délit qui reformule, en les diluant, toutes ces frontières entre des prisonnières qui se rangent maintenant dans la nouvelle macro-catégorie emic désignée par " pour drogue ». C'est que dans leur vie pré-carcérale ces catégories étaient déjà mélangées, dans le contexte de la famille, pour commencer. Ainsi, et pour en donner un exemple qui est fréquent, une trafiquante non consommatrice a un mari consommateur (qui est ou n'est pas trafiquant) et un fils toxicomane qui, à un moment donné, a volé des affaires dans la maison pour les vendre.

Cette conjonction pré-carcérale de catégories carcérales est d’ailleurs homologue et cohérente avec celle que l'on peut constater dans un autre champ de la vie des prisons. En effet, si, il y a dix ans, la crainte d'être contaminé par des maladies comme l'hépatite $B$, mais surtout le sida, était très vive et tout à fait appropriée pour exprimer physiquement et métaphoriquement la distance et la non-identification entre les détenues, aujourd'hui ce n'est plus le cas. Les précautions prises, quand elles sont prises, sont d'ordre strictement sanitaire et n'ont aucun contenu moral (je n'ai jamais entendu un mot ou observé un comportement distanciateur, par exemple). Il y a même une préoccupation en sens inverse : il s'agit de ne pas contaminer, par exemple par un rhume, une détenue séropositive dont le système immunitaire est fragilisé. Encore une fois, il s'agit d'une réalité qui est souvent proche avant la détention : elles ont un fils ou un frère qui a le sida.

D’autres frontières encore se reformulent, deviennent perméables ou se brouillent. Ce sont des frontières articulées à des niveaux d'identité tels que le quartier d'origine, la « race » et l'ethnicité. Le quartier d'origine est un référent identitaire important ; il est identifié comme lieu d'appartenance et peut même être codé par un tatouage spécifique ${ }^{4}$. L'appartenance 
commune à un quartier est un instrument d'intégration important au début de l'incarcération : une nouvelle arrivée est aidée et initiée aux règles formelles et informelles du fonctionnement de la prison par d'autres détenues du même quartier, indépendamment du fait qu'elles se connaissaient ou non avant la détention. Pourtant, cet alignement social par quartiers se dilue très vite et les groupes se forment selon une logique de sociabilité qui ne prend pas en compte ces catégories d'insertion. Ce mélange d'ailleurs n'est pas nécessairement un phénomène d'ordre carcéral. À l'extérieur, ces quartiers périurbains ne sont pas des ghettos sociaux et identitaires. Par exemple, il existe entre eux une forte circulation de personnes qui se fait moins en raison des réseaux de l'économie illégale, qu'en fonction des liens de parenté, d'amitié ou de travail.

D’autres référents identitaires passent par des marqueurs raciaux ou ethniques exprimés par les mots «blanche ", " noire », " gitane », " corrilha » (" non gitane »), " cap-verdienne », « angolaise » et "africaine ». Ces référents ne sont pas très présents dans le discours quotidien des détenues. Ils ont très peu d'implications dans les pratiques de sociabilité, et surtout ne font pas l'objet de manipulations d'ordre identitaire, par exemple par une catégorie qui viserait à dénigrer ou à négativiser collectivement une autre. Ce type de déqualification est produit principalement par une minorité de détenues de couches sociales plus favorisées (elles sont en général blanches) et dont le crime n'a aucun lien avec la drogue. Ce sont surtout celles-ci qui procèdent à une catégorisation amalgamant drogue, quartier ghetto, race ou ethnie et dégradation. Cet amalgame évoque d'ailleurs des représentations émergeant à l'extérieur de la prison, où elles construisent de nouvelles « classes dangereuses » [Valentim, 1997]. Ces référents raciaux ou ethniques ne délimitent donc pas de catégories opérant au quotidien. Leur seule valeur est indicative. D’ailleurs, l'unique contexte dans lequel j'ai vu cette valeur en action a été celui de l'interaction des détenues avec moi, qui suis extérieure à cet univers mélangé. Elles pouvaient dire, par exemple, que telle copine est « blanche comme vous » ou de « votre race».

Une fois encore, les frontières qui, il y a dix ans, délimitaient des clivages tangibles, se sont aujourd'hui beaucoup diluées. J’ai déjà signalé le nivellement social qui se fait par le bas de la grande majorité des détenues, une semblable homogénéisation concernant aussi le type de crime. De plus, avant la prison, les détenues de ces différentes catégories partageaient aussi des modes de vie proches, des trajectoires de vie similaires, des occupations semblables dans l'économie légale et illégale, et souvent vivaient dans les mêmes quartiers. C'est comme si au long de ces dix ans s’était opérée, intra- et extra-muros, une intégration dans l'exclusion.

Inversement, tandis que les frontières séparant les détenues se brouillent, le personnel pénitencier a tendance a amplifier la distance qui le sépare d'elles en exotisant la pauvreté. Ceci s'exprime par le thème, entre autres, de la " misère morale » qui accompagne la misère matérielle, ou par le thème d'une mentalité spécifique et incompréhensible, par exemple au travers de la question qui est fréquemment posée : "Comment peut-on trafiquer de la drogue quand on a un fils toxicomane ? " Il n'est pas rare que la différence sociale soit transformée ou pensée en tant que différence culturelle. Par exemple, une éducatrice parlant de la sympathie qu'elle ressentait pour les détenues gitanes me faisait part de sa perplexité et se demandait pourquoi les gitans vivaient toujours dans des «baraques» ou dans des bidonvilles. Et elle concluait : «Ça doit être culturel. »

Tandis que les clivages divisant les diverses catégories de détenues se gomment à présent, ils semblent en revanche se creuser à l'intérieur de chaque catégorie. C'est tout d'abord sensible dans l'incorporation de représentations hégémoniques s'exprimant à l'égard d'une catégorie 
par les membres de cette catégorie elle-même. Ainsi, il n'est pas rare qu'une gitane évite d'autres gitanes en faisant appel à des stéréotypes comme "les gitans ne se lavent pas » ou "on ne peut pas faire confiance à un gitan». Il s'agit ensuite de clivages d'ordre générationnel. D’une part, et surtout en ce qui concerne les gitanes et les détenues d'origine africaine, la génération plus jeune est déjà impliquée dans la consommation de drogues, ce qui arrive rarement avec les mères qui se limitent au trafic (les pères étant plus souvent consommateurs). D’autre part, ces clivages de génération, cette fois toutes catégories confondues, se manifestent dans un ethos et un style plus réactifs et oppositionnels de la part des plus jeunes, une affirmation positive de ce que l'on peut appeler une « culture de rue ».

Malgré ce nouveau type de clivages, qui ne définissent pourtant pas de nouvelles catégories, les frontières marquées qui étaient à l'œuvre lors de ma première enquête se sont globalement dissoutes. Il n'aurait alors pas été possible d'entendre, comme aujourd'hui, "on est toutes ensemble » ou " on est toutes dans le même bateau ». Mon extériorité à cet univers était hier un avantage, il est aujourd'hui un obstacle. Hier, le rapprochement rapide qui s'était opéré entre les détenues et moi-même s'inscrivait dans le mouvement de distanciation qui les séparait. Aujourd'hui ce mouvement n'est plus entrepris (sauf par une minorité) et il m'a fallu beaucoup plus de temps pour construire une relation de proximité. Cependant, mes difficultés actuelles s'articulent sur un deuxième ordre de raisons ou de barrières. Ou, plus précisément, ces difficultés se comprennent aussi par la proximité qui lie les détenues. Mais cette fois il ne s'agit pas d'une proximité sociologique et symbolique, mais de la proximité très concrète, faite de réseaux d'inter-connaissance, de relations de parenté et de voisinage. Cette proximité présente aussi le revers de comporter un important potentiel de tension et de conflit. Tout d'abord, bien que parfois bruyants et étalés devant les co-détenues, ces tensions et ces conflits sont l'objet d'une grande discrétion devant l'ethnographe, parce qu'ils relèvent de l'ordre familial, donc privé. Mais celui-ci ne reste plus, comme autrefois, à l'extérieur de la prison. En voici un exemple, pris parmi de nombreux autres.

Deux détenues sont placées à leur demande dans la même cellule, car elles étaient voisines dans le même quartier; à l'extérieur, la sœur de l'une commence à vivre avec le mari de l'autre, dont elle a un fils ; la cohabitation des prisonnières devient très problématique, et elles se séparent. Il est arrivé aussi dans d'autres cas que la nouvelle compagne du mari soit emprisonnée; la prudence recommande alors que les deux détenues soient logées non seulement dans des cellules séparées, mais même dans des pavillons différents. Comme chacune a des membres de sa famille dans l'établissement, et comme les familles se connaissent à l'extérieur, on entrevoit la délicatesse dont il faudra faire preuve pour aborder, dans les entretiens, quelque sujet que ce soit qui touche aux relations internes et principalement la question, si intrigante au début, des transferts constants de cellule et de pavillon. Le linge de la maison, c'est-à-dire ce qui est de l'ordre du privé, peut encore se laver devant les voisins, mais pas devant une personne presque inconnue. Des tensions carcérales peuvent donc avoir une origine extra-carcérale (le contraire est aussi avéré) et sont a priori incluses dans la sphère privée.

Ensuite, si la discrétion est de rigueur dans le domaine des affaires de famille, le silence est vital dans un autre domaine. Il s’agit de l'enjeu pénal, souvent lui aussi extra- ou prépénitentiaire. En règle générale, beaucoup de négociations ont lieu entre frères, belles-sœurs, cousines (c'est-à-dire dans un cercle familial pouvant être très élargi et même inclure des voisins), de façon à ce que l'un d'eux assume la responsabilité du délit et que les autres accusés puissent rester en liberté ou être libérés. Il est impératif alors que la détenue garde le silence, ce qui n'arrive pas toujours, notamment si elle ne se sent pas assez soutenue par des 
visites ou des colis. Néanmoins, l'impératif de la non-délation est à présent beaucoup plus fort qu'il y a dix ans. Il concerne en effet désormais moins les activités clandestines dans la prison que l'illicite pénal à l'amont de la prison, dont les conséquences sont plus lourdes. De plus, les personnes protégées par le silence sont aujourd'hui des proches et non de simples co-détenues. Enfin, cet impératif de non-délation est aussi déjà pré-carcéral, autrement dit il est cultivé dans le contexte des réseaux de solidarité de voisinage et familiale. Néanmoins ces valeurs de solidarité et de non-délation peuvent entrer en contradiction et elles sont alors constamment re-hiérarchisées. Deux exemples l'attestent. Une détenue A a une fille en liberté qui est trafiquante de drogue ; cette fille finit par être incarcérée préventivement, mais avec une tante qui, elle, n'en a jamais vendu un gramme. Cette dernière est même l'une de ces héroïques résistantes à qui j'ai déjà fait allusion : elle a pris deux emplois, un de jour et un de nuit, pour que son fils, toxicomane, n’ait pas à voler. Pour protéger sa nièce elle ne dit rien. Mais une détenue $B$, sœur de cette tante, ne trouve pas juste que sa sœur innocente soit condamnée et elle entre en conflit avec la détenue A, laquelle voudrait que sa fille continue à bénéficier du silence généreux de sa tante. L'autre exemple concerne une prisonnière qui domine assez bien les réseaux de son quartier. Elle a un fils toxicomane qui lui a volé des affaires dans sa maison et les a vendues à une voisine, qui elle-même entretient des contacts avec l'univers du trafic. Or, quand il est pratiqué dans le même quartier et quand il concerne des biens appartenant aux personnes de ce quartier, le recel est particulièrement censuré parce qu'il met en cause l'expectative de solidarité de voisinage. Extrêmement choquée, cette détenue envisageait de dénoncer le trafic que pratiquait la voisine.

Liant ainsi de façon quotidienne et inextricable la prison à l'extérieur, toutes ces tensions sont l'objet d'une discrétion et d'une réserve accrues, d'autant plus que tout événement carcéral peut avoir des répercussions extra-muros dont les effets seront toujours là une fois la peine terminée. Comment penser alors la prison ? Entre l' " institution totale » goffmanienne et la «micro-société »/« micro-culture » ${ }^{5}$, le statut théorique de la prison s'est bâti sur la notion que ses limites matérielles délimitaient un monde à part. Il ne restait qu’à formuler ce monde empiriquement. Comme autrefois on étudiait " un village », dans la prison aussi « terrain » et « objet » se confondaient. Aujourd'hui, de ce point de vue, ce " terrain » est moins " miné ». Les questions s'y font plus précises et constituent des problématiques plus spécifiques. Mais la prison en tant qu'unité d'observation donnée, se prêtant à l'enquête de terrain, reste encore l'unité privilégiée d'analyse, même si l'horizon s'élargit et si on cherche les processus historiques et globaux qui aident à éclairer des logiques internes.

Il y a dix ans, cette dernière approche se révélait relativement adéquate à l'univers que j'étudiais. Les frontières physiques de cette prison - qui étaient aussi, on l'a vu, des frontières symboliques - traçaient également "un cadre de vie temporaire spécifique, doté d'une relative autonomie, et un cadre de relations sociales aux dynamiques propres " [Cunha, 1995 : 120], même si les éléments de leur interprétation n’étaient pas entièrement contenus intra-muros [Cunha, 1994]. Or aujourd'hui, bien que la traversée de ces frontières ait des conséquences réelles dans la vie de ces femmes et de leurs proches, notamment de leurs enfants, sa portée sociologique s'est réduite. La continuité intérieur-extérieur est à présent telle que la prison semble ne plus pouvoir être pensée que dans la marge et le mouvement entre les deux. La principale «mine », si l'on veut, est que les murs de la prison cachent leur porosité.

\section{Notes}




\section{Références bibliographiques}

ALBINI Joseph, 1992, "The Distribution of Drugs : Models of Criminal Organization and their Integration », in Mieczkowski Tom (sous la dir. de), Drugs, Crime and Social Policy : Research, Issues and Concerns, Boston, Allyn and Bacon : 79-108.

BARRÉ M.D., 1996, «Toxicomanie et délinquance : relations et artefacts », Déviance et Société, 4 (4).

Chaves Miguel, 1999, Casal Ventoso: da Gandaia ao narcotráfico. Marginalidade económica e dominação simbólica num bairro de Lisboa, Lisbonne, Imprensa de Ciências Sociais.

CunHA Manuela, 1991, "Investigar 'problemas sociais': equívocos e dilemas de uma etnografia da prisão », Cadernos do Noroeste, 4 (6-7) : 193-209.

— 1994, "Malhas que a reclusão tece : questões de identidade numa prisão feminina », Cadernos do CEJ, 2/92.

- 1995, «Sociabilité, 'société', ‘culture’ carcérales: la prison féminine de Tires (Portugal) », Terrain, 24 : 119-132.

DoRn Nicholas, Karim MurJi, Nigel SouTH, 1992, Traffickers : Drug Markets and Law Enforcement, Londres, Routledge.

KENNA Margaret, 1992, "Changing places and altered perspectives : research on a Greek island in the 1960s and in the 1980s », in Judith Okely, Helen Callaway (sous la dir. de), Anthropology and Autobiography, Londres, Routledge : 147-162.

OKELY Judith, 1992, «Anthropology and Autobiography: Participatory Experience and Embodied Knowledge », in Judith Okely, Helen Callaway (sous la dir. de), Anthropology and Autobiography, Londres, Routledge : 1-28.

Reuters P., 1983, Disorganised Crime : Illegal Markets and the Mafia, Cambridge (Mass.), MIT Press.

SMITH David J., 1997, «Ethnic Origins, Crime and Criminal Justice », in M. Maguire, R. Morgan, R. Reiner, The Oxford Handbook of Criminology, Oxford, Clarendon Press.

VALENTIM Artur, 1997, «A construção social do problema-Droga em Portugal: alguns dados sobre a evolução recente », Análise Social, 25 : 82-102.

\section{ABSTRACT}

Ways and by-ways around a women's prison

The prison constitutes one of the fields of research which, immediately and by general agreement, seem to be covered by the notion of "minefield", in particular because the context has already been defined as a "social problem" and because the definition of a research project is not always easy. But there are other mines, traps, and theoretical as well as methodological questions not initially apparent which are not different from those existing in 
virtually all other fields. That is the type of mine we are dealing with here. The article describes a return to the original site after ten years and examines the problems which have beset a more recent research project. The profound changes which have marked the penal institutions for women in Portugal are presented and the discussion of the theoretical state of prisons and their borders on the outside worlds is re-opened.

Keywords : Prison. Women. Drugs. Exclusion. Portugal.

\section{ZUZAMMENFASSUNG}

Wege und Umwege um ein Frauengefängnis

Das Gefängnis stellt einen jener Forschungsbezirke dar, die von vornherein und ohne Diskussion als "Minenfeld" eingestuft werden könnten, insbesondere, weil dieser Bezirk ausserdem als "soziales Problem" vorgeprägt und die Erstellung eines Forschungsziels nicht immer selbstverständlich ist. Daneben bestehen jedoch weitere Minen, Fallen, sowie anfangs nicht erkennbare theoretische und methodologische Fragen, die sich nicht von denen unterscheiden, die sich praktisch auf allen Gebieten finden. Um diese Art Minen handelt es sich hier. Der Artikel behandelt eine Situation der Rückkehr an den Ort des Geschehens nach zehn Jahren und beleuchtet die Schwierigkeiten, die die Durchführung einer neueren Untersuchung gekennzeichnet haben. Die tiefgreifenden Veränderungen, die eine portugiesische Frauenstrafanstalt erfahren hat, werden herausgearbeitet und die Diskussion über das theoretische Statut des Gefängnisses und über seine Grenze zur äusseren Welt wird erneut aufgeworfen.

Stichwörter : Gefängnis. Frauen. Drogen. Ausgrenzung. Portugal.

\footnotetext{
* Je remercie la Wenner-Gren Foundation for Anthropological Research, qui a financé une partie de cette recherche.

${ }^{1}$. Néanmoins, la proportion de femmes dans la population pénitentiaire du Portugal est passée de $6 \%$ à 9,5 \% .

2 . Je me limite ici à signaler cette relation sans m'étendre sur la nature du rapport existant entre drogues et délinquance. Il s'agit en effet d'une relation complexe, et aussi bien le sens de cette corrélation que les directions de causalité entre l'une et l'autre nourrissent un débat qui a presque trente ans déjà [Barré, 1996].

3 . Sans examiner en détail la constitution et le fonctionnement de ces réseaux, je signale qu'il s'agit plutôt de nombreux groupes de petite dimension, avec une structure flexible et fonctionnant de façon autonome (quelques auteurs parlent de « crime désorganisé » [Dorn et al, 1992 ; Reuters, 1983]), et non de quelques grands réseaux, centralisés, hiérarchisés et comportant un grand nombre de personnes [Albini, 1997 ; Chaves, 1999].
}

4 . Cependant, ce même signe peut être tatoué sur des détenues provenant d'autres quartiers, et il est alors strictement décoratif.

5 . La « société carcérale » et la « culture pénitentiaire » ont été des thèmes centraux qui ont traversé l’histoire de la recherche sur les prisons [Cunha, 1995]. 